\title{
Maternal Age/Delivery Times-specific Reference Intervals of Fibrin Related Biomarkers During Normal Late Pregnancy in Han Population from Southwest China
}

\author{
Liu Dinghua ", Liu Huan, He Qi, Li Xin, Cai Wei \\ Department of Clinical Laboratory, University-Town Hospital of Chongqing Medical University, Chongqing, China \\ Email address: \\ dinghualiu@cqmu.edu.cn (Liu Dinghua) \\ ${ }^{*}$ Corresponding author
}

\section{To cite this article:}

Liu Dinghua, Liu Huan, He Qi, Li Xin, Cai Wei. Maternal Age/Delivery Times-specific Reference Intervals of Fibrin Related Biomarkers During Normal Late Pregnancy in Han Population from Southwest China. International Journal of Clinical and Experimental Medical Sciences. Vol. 6, No. 3, 2020, pp. 46-50. doi: 10.11648/j.ijcems.20200603.14

Received: June 9, 2020; Accepted: June 23, 2020; Published: June 29, 2020

\begin{abstract}
Objective: Human pregnancy requires hypercoagulation of blood, which means haemostatic reference intervals based on normal people may not be suitable for pregnant women. The aim of this study was to establish reference intervals of fibrin related biomarkers during normal late pregnancy in Han population from southwest China. Methods: Plasma samples were collected from 2374 healthy pregnant women (35-40 gestational weeks) and 326 healthy non-pregnant women at University-Town Hospital of Chongqing Medical University from January 2017 to December 2019. All of the subjects were assigned to 4 groups by age: A (18-29 years), B (30-34 years), C (35-39 years) and D (40-45 years). Further, the pregnant subjects were assigned to 2 groups by delivery times: I (first-delivery) and II (second-delivery). Fibrinogen (Fbg), D-Dimer and fibrin (-ogen) degradation products (FDP) were measured by Sysmex CS-5100 coagulation analyzer. Results: The Fbg reference intervals during late pregnancy in local Han population were $4.09 \pm 1.12 \mathrm{~g} / \mathrm{L}$. The D-Dimer reference interval for first-delivery pregnancy was $<2.77 \mathrm{mg} / \mathrm{L}$, and those of second-delivering women were $<3.37 \mathrm{mg} / \mathrm{L}(18-34$ years) and $<3.99 \mathrm{mg} / \mathrm{L}$ ( $35-45$ years). The FDP reference interval for first-delivery pregnancy was $<9.28 \mathrm{mg} / \mathrm{L}$, and those of second-delivering women were $<10.52 \mathrm{mg} / \mathrm{L}(18-34$ years) and $<11.72 \mathrm{mg} / \mathrm{L}$ (35-45 years). Conclusion: In the current study, maternal age/delivery times-specific reference intervals of plasma Fbg, D-Dimer and FDP during normal late pregnancy in Han population from southwest China were presented, which could assist in clinical diagnosis and treatment. Furthermore, we demonstrated maternal age and delivery times did significantly affect the plasma levels of D-dimer and FDP in normal late pregnant women.
\end{abstract}

Keywords: Fibrin-related, Late Pregnancy, Reference Interval, Second Delivery

\section{Introduction}

With the implementation of comprehensive two-child policy, since late 2015, the amount of pregnant women aged over 35 years in China has been rising apparently [1]. Although there is no direct evidence to prove that the new policy should be responsible for the increasing maternal morbidity or mortality, obstetrics resources do become scarcer and maternal ages do become more advanced in our country, which give the rise to high risk of maternal and fetal health. Due to the physiological need of pregnancy and delivery, changes in all aspects of haemostatic system result in a shift of haemostatic balance to hypercoagulability, which protects pregnant women from serious bleeding complications at delivery [2]. But on the other hand, hypercoagulability of blood combining with venous stasis and endothelial damage during pregnancy and puerperium create an incidence of venous thromboembolism (VTE) 4-50 times higher than non-pregnant women especially in the period of late pregnancy and puerperium [3-4]. Therefore, sensitive, reliable and less-invasive experimental biomarkers would value a lot in VTE diagnosis and treatment for pregnant women.

Up to date, fibrin-related biomarkers of coagulation and fibrinolytic system, which are commonly tested and relatively standardized in clinical laboratory, include 
fibrinogen (Fbg), D-Dimer and fibrin (-ogen) degradation products (FDP) [5-7]. Accurate testing results and proper reference intervals provide effective evidences for clinical decision. Based on the above-mentioned physiological changes, plasma fibrin-related biomarkers of pregnant women increase more or less compared with non-pregnant healthy women. Meanwhile, owing to different locations, environments, population ethnicities and laboratory conditions, differences do exist to some extend among the results of the same specimen. All of the above-mentioned raise the necessity to establish specific reference intervals of fibrin-related biomarkers for healthy pregnant women. Therefore, based on our previous work, we recruit more subjects, differentiate maternal age and include second-childbirth women in the current study to establish maternal age/delivery times-specific reference intervals of fibrin related biomarkers during normal late pregnancy in Han population.

\section{Data and Methods}

\subsection{General Data}

A total of 4189 healthy women undergoing late pregnancy from January 2017 to December 2019 in our hospital were observed consecutively. After the duration of pregnancy, 2374 pregnant women were enrolled in the study finally. Inclusion criteria were [8]: han population; natural singleton pregnancy; aged 18-45 years; 35-40 gestational weeks; full-term living birth; no complication or disease during pregnancy and delivery. Exclusion criteria were: history of abortion; personal or family history of thyroid disease or other autoimmune diseases; medical history of thrombosis and thrombolysis; aspirin, heparin or other anti-coagulation or fibrinolysis treatment during pregnancy and delivery. Additionally, 326 healthy non-pregnant women aged $18-45$ years were included as control group under the same exclusion criteria. All of the subjects were assigned to 4 groups by age: A (18-29 years), B (30-34 years), C (35-39 years) and D (40-45 years). Furthermore, the pregnant subjects were assigned to 2 groups by delivery times: I (first-delivery) and II (second-delivery).

\subsection{Methods}

\subsubsection{Sample Collection and Processing}

Two point seven $\mathrm{mL}$ of blood were collected by vein-puncture into vaccum tubes containing $0.109 \mathrm{~mol} / \mathrm{L}$ sodium citrate. After immediate and gentle mixing, blood samples were centrifuged at $3000 \times \mathrm{g}, 20^{\circ} \mathrm{C}$ for $10 \mathrm{~min}$ to collect plasma. Plasma samples were tested in $2 \mathrm{~h}$ after vein-puncture.

\subsubsection{Laboratory Measurement}

All assays of Fbg, D-Dimer and FDP were performed on CS-5100 automated coagulation analyzer (Sysmex, Japan). Reagents, calibrators and quality controls were original products by Sysmex. All measurements were conducted according to the standard operating procedures of the instrument and the tests. The characteristics of the tests performed were shown in Table 1.

Table 1. Characteristics of the tests performed.

\begin{tabular}{ll}
\hline Test & Method \\
\hline Fbg & Clauss assay \\
D-Dimer & Particle-enhanced immuno-turbidimetric assay \\
FDP & Particle-enhanced immuno-turbidimetric assay \\
\hline
\end{tabular}

\subsection{Statistical Methods}

Statistical analyses were performed using SPSS 20.0 software. The data were reported as mean or median valves depending on the distribution type of data by Kolmogorov-Smirnov test. Statistical comparisons were tested with T test, Mann-Whitney test and Kruskal-Wallis test. And $P<0.05$ indicates significant difference.

\section{Results}

Data shown in Tables 2, 3, 5 demonstrated that three fibrin-related biomarkers levels of pregnant women with different ages or delivery times were significantly higher than controls, respectively $(P<0.05)$.

\subsection{Fbg}

Normal-distributing data of Fbg were described by mean and SD in Table 2. Based on that both low and high level of Fbg were considered abnormal in clinical practice, two-tail reference interval of Fbg was described by mean $\pm 2 \mathrm{SD}$. Delivery times-specific differences of plasma $\mathrm{Fbg}$ in late pregnant women with the same age were not significant, and age-specific differences of Fbg levels with the same delivery times were not significant either $(P>0.05)$. Therefore, it was appropriate to establish reference interval of $\mathrm{Fbg}$ for late pregnant women regardless of maternal age and delivery times (Table 2).

Table 2. Measurements of Fbg and reference interval in late-pregnant women.

\begin{tabular}{llllll}
\hline subject & $\mathbf{n}$ & Fbg $(\mathbf{g} / \mathbf{L})$ & control & $\mathbf{n}$ & Fbg (g/L) \\
\hline AI & 1216 & $3.95 \pm 0.63^{\Delta}$ & A & 125 & $2.44 \pm 0.50$ \\
BI & 298 & $3.91 \pm 0.30^{\Delta}$ & & & \\
CI & 75 & $4.23 \pm 0.33^{\Delta}$ & B & 83 & $2.51 \pm 0.44$ \\
DI & 50 & $4.25 \pm 0.64^{\Delta}$ & & & \\
AII & 287 & $3.88 \pm 0.65^{\Delta}$ & $\mathrm{C}$ & 66 & $2.82 \pm 0.58$ \\
BII & 243 & $3.90 \pm 0.68^{\Delta}$ & & & \\
CII & 152 & $4.32 \pm 0.59^{\Delta}$ & $\mathrm{D}$ & 52 & $2.62 \pm 0.46$ \\
DII & 53 & $4.41 \pm 0.90^{\Delta}$ & & & \\
\multicolumn{5}{l}{ Fbg reference interval: $4.09 \pm 1.12 \mathrm{~g} / \mathrm{L}(\mathrm{n}=2374)$} \\
\hline
\end{tabular}

$\Delta$ : compared with control group, $P<0.05$.

\subsection{D-Dimer}

Skewed-distributing data of D-Dimer were described by median, 25th and 75th percentiles $\left(\mathrm{M}\left(\mathrm{P}_{25}, \mathrm{P}_{75}\right)\right)$ in Table 3 . According to clinical use of that high level of D-Dimer was considered abnormal, one-tail reference interval of D-Dimer was described by 95 th percentile $\left(<\mathrm{P}_{95}\right)$. Data indicated that most differences of D- Dimer levels between group I and II 
with the same age were significant, hence it was proper to establish specific reference intervals of D-Dimer for pregnant women who delivered firstly or secondly, respectively. Table 3 showed that there were no significant differences of D-Dimer levels according to different ages in group I $(P>0.05)$, so reference interval of D- Dimer for first-delivering women should be established regardless of age (Table 4). Based on the significant differences between group CII and AII/BII, group DII and AII/BII $(P<0.05)$, it was necessary to establish D-Dimer reference intervals differentiated by age 35 for second-delivering women (Table 4).

Table 3. Measurements $f$ D-Dimer in late-pregnant women.

\begin{tabular}{llllll}
\hline subject & $\mathbf{n}$ & D- Dimer $(\mathbf{m g} / \mathbf{L})$ & control & $\mathbf{n}$ & D- Dimer $(\mathbf{m g} / \mathbf{L})$ \\
\hline AI & 1216 & $1.36(1.03,2.03)^{\Delta}$ & $\mathrm{A}$ & 125 & $0.15(0.07,0.22)$ \\
BI & 298 & $1.64(1.06,2.21)^{\Delta}$ & & & \\
CI & 75 & $1.41(0.91,2.15)^{\Delta}$ & B & 83 & $0.18(0.13,0.28)$ \\
DI & 50 & $1.06(0.82,2.28)^{\Delta}$ & & & \\
AII & 287 & $1.64(1.15,2.35)^{\Delta^{*}}$ & C & 66 & $0.25(0.16,0.35)$ \\
BII & 243 & $1.73(1.17,2.28)^{\Delta}$ & & & \\
CII & 152 & $2.34(1.53,3.87)^{\Delta^{* \#}}$ & D & 52 & $0.13(0.08,0.29)$ \\
DII & 53 & $2.88(1.52,3.94)^{\Delta^{* \#}}$ & & & \\
\hline
\end{tabular}

$\Delta$ : compared with control group, $P<0.05$; *: compared between group I and II for the same age interval, $P<0.05$; \#: compared with group BII and AII, $P<0.05$.

Table 4. D-Dimer reference intervals of late-pregnant women.

\begin{tabular}{llll}
\hline & $\mathbf{n}$ & $\mathbf{M}\left(\mathbf{P}_{\mathbf{2 5}}, \mathbf{P}_{\mathbf{7 5}}\right)$ & reference interval $<\mathbf{P}_{\mathbf{9 5}}$ \\
\hline first-delivery & 1639 & $1.59(1.13,2.29)$ & 2.77 \\
second-delivery & & & \\
18-34 years & 530 & $1.69(1.14,2.37)$ & 3.37 \\
35-45 years & 205 & $2.66(1.51,3.88)$ & 3.99 \\
\hline
\end{tabular}

\subsection{FDP}

Skewed-distributing data of FDP were also described by median, 25th and 75th percentiles $\left(\mathrm{M}\left(\mathrm{P}_{25}, \mathrm{P}_{75}\right)\right)$ in Table 5 . According to clinical use of that high level of FDP was considered abnormal, one-tail reference interval of FDP was described by 95 th percentile $\left(<\mathrm{P}_{95}\right)$. Data indicated that the differences of FDP levels between group I and II with the same age were significant, so it was rational to establish specific reference intervals of FDP for pregnant women who delivered firstly or secondly, respectively. It is shown in Table 5 that there were no significant differences of FDP levels regarding to different ages in group I $(P>0.05)$, so FDP reference interval of first-delivering women should be established regardless of age (Table 6). Based on the significant differences between group $\mathrm{CII}$ and $\mathrm{AII} / \mathrm{BII}$, group DII and AII/BII $(P<0.05)$, it is necessary to establish FDP reference intervals differentiated by age 35 for second-delivering women (Table 6).

Table 5. Measurements of FDP in late-pregnant women.

\begin{tabular}{llllll}
\hline subject & $\mathbf{n}$ & FDP $(\mathbf{m g} / \mathbf{L})$ & control & $\mathbf{n}$ & FDP $(\mathbf{m g} / \mathbf{L})$ \\
\hline AI & 1216 & $4.22(3.33,5.70)^{\Delta}$ & $\mathrm{A}$ & 125 & $1.02(0.81,1.33)$ \\
BI & 298 & $4.35(3.64,6.81)^{\Delta}$ & & & \\
CI & 75 & $4.13(2.91,5.84)^{\Delta}$ & B & 83 & $1.08(0.63,1.61)$ \\
DI & 50 & $4.40(3.13,5.92)^{\Delta}$ & & & \\
AII & 287 & $4.72(3.55,6.51)^{\Delta^{*}}$ & C & 66 & $1.12(1.03,1.23)$ \\
\hline
\end{tabular}

\begin{tabular}{llllll}
\hline subject & $\mathbf{n}$ & FDP $(\mathbf{m g} / \mathbf{L})$ & control & n & FDP $(\mathbf{m g} / \mathbf{L})$ \\
\hline BII & 243 & $4.95(3.38,6.30)^{\Delta^{*}}$ & & & \\
CII & 152 & $7.82(4.54,8.87)^{\Delta^{* \#}}$ & D & 52 & $1.11(0.88,1.43)$ \\
DII & 53 & $6.14(3.15,9.53)^{\Delta^{* \#}}$ & & & \\
\hline
\end{tabular}

$\Delta$ : compared with control group, $P<0.05 ; *$ : compared between group I and II for the same age interval, $P<0.05$; \#: compared with group BII and AII, $P<0.05$.

Table 6. FDP reference intervals of late-pregnant women.

\begin{tabular}{llll}
\hline & $\mathbf{n}$ & $\mathbf{M}\left(\mathbf{P}_{\mathbf{2 5}}, \mathbf{P}_{\mathbf{7 5}}\right)$ & reference interval $<\mathbf{P}_{\mathbf{9 5}}$ \\
\hline $\begin{array}{l}\text { first-delivery } \\
\text { second-delivery }\end{array}$ & 1639 & $4.70(3.51,6.72)$ & 9.28 \\
18-34 years & 530 & $4.93(3.14,6.62)$ & 10.52 \\
35-45 years & 205 & $6.84(3.33,9.14)$ & 11.72 \\
\hline
\end{tabular}

\section{Discussion}

Throughout pregnancy, thrombin generation and following fibrinolysis increase, resulting in elevation of coagulation and activation of fibrinolysis, which are considered physiological for maintaining placental function during pregnancy and protecting women from haemorrhage at delivery [9]. But the physiological pregnancy-induced hypercoagulation as well as decreased venous outflow of lower extremities may contribute to thrombosis and high incidence of VTE at the same time [10]. Previous studies reported the incidence of VTE in pregnant women 4-50 higher than non-pregnant age matched women and 1.72 per 1000 deliveries with acute pulmonary embolism [3-4]. Since late 2015 with implementation of the new two-children policy, it was initially estimated that $60-70$ percent of women delivering second child aged over 35 years [1]. Advanced maternal age leads to much higher risk of haemostatic complications during pregnancy, delivery and puerperium, especially in those women who gave the first birth by cesarean section or developed hypertension, diabetes mellitus, pre-eclampsia and so on during the first pregnancy [11]. Consequently, physicians need to fulfill diagnosis to rule out of VTE in late-pregnant woman as soon as possible, which requires sensitive, reliable and low-invasive biomarkers [12].

Fibrin related markers being extensively tested among hospitals at different levels in China include Fbg, D-dimer and FDP. Fbg, a coagulation parameter, is widely used to monitor the status of hypercoagulation or hyperfibrinolysis in human body. D-dimer, a secondary-fibrinolysis parameter, is very helpful in ruling out VTE due to its high negative predictive value in clinical decision in case of low probability pre-test Wells scores. FDP, representing a series of products of fibrinolysis, is usually used with D-Dimer to distinguish primary and secondary hyperfibrinolysis. According to the above-mentioned pregnancy-induced changes, the levels of Fbg, D-dimer and FDP increase a lot during pregnancy. Therefore, reference intervals of Fbg, D-dimer and FDP based on non-pregnant healthy women are inappropriate for clinical decisions of pregnant women. Meanwhile, due to differences of regions, populations, life habits and testing assays, reference interval of biomarker differs to some extend, which requires clinical laboratory to build or validate reference interval of every biomarker. 
In the present study, we enrolled more subjects with different ages (especially advanced maternal ages) and compared data of first-delivery pregnant women to second-delivery ones, in order to establish reference intervals of Fbg, D-dimer and FDP by enough data. In accordance with CLSI C28-A3 [13] and under proper inclusive and exclusive criteria, we analyzed 2374 late pregnant healthy Han women (1639 first-delivery and 735 second-delivery). Our data demonstrated that Fbg, D-dimer and FDP levels of late pregnant women increased remarkably compared with those of age-matched control subjects, hence specific reference intervals of plasma Fbg, D-dimer and FDP for these populations should be established for the sake of accurate medical decisions.

Data of the current study showed normal distribution of Fbg and skewed distribution of D-Dimer and FDP. According to clinical usage in practice, abnormality of Fbg is defined with too low or too high level, while that of D-Dimer or FDP is with too high level, therefore $95 \%$ two-tail reference interval was calculated for Fbg and 95th percentiles were calculated for one-tail reference intervals of D-Dimer and FDP. As to pregnant women, maternal age and delivery times should also be estimated when establishing reference interval. Our study assigned all of the subjects to 4 groups by maternal age and further to 2 groups by delivery times. Data showed that Fbg reference interval of late pregnant women should be established regardless of maternal age and delivery times. Based on comprehensive analysis of D-dimer and FDP data, reference intervals of D-Dimer and FDP for first-delivery women should be established regardless of maternal age, while those for second-delivery women should be differentiated by age 35 .

Our reference intervals of plasma Fbg, D-dimer and FDP were not similar to those being reported more or less [14-16], which might be attributed to regions, populations, testing assays, instruments and reagents, etc, requiring clinical laboratories to confirm their own reference intervals of plasma Fbg, D-dimer and FDP by establishing or validating. Clauss assay for $\mathrm{Fbg}$ and particle-enhanced immunoturbidimetric assay for D-Dimer/FDP run tests fast and withstand interference well. Both of the assays are applied in majority of clinical laboratories around the world. Therefore, using these assays to establish reference intervals of plasma Fbg, D-dimer and FDP would be more practical and reproducible.

However, some limitations of this study should be noted. Firstly, due to practical conditions, first and second-trimester pregnant women have not been studied yet. Secondly, established reference intervals need to be validated with enough positive cases. All of the mentioned raised the need for our further study in future.

\section{Conclusion}

In conclusion, we presented reference intervals of fibrin related biomarkers (Fbg, D-dimer and FDP) during normal late pregnancy in Han population with natural singleton pregnancy, which could provide reliable evidences for accurate diagnosis and effective treatment, and further we demonstrated maternal age and delivery times did significantly affect the plasma levels of D-dimer and FDP in normal late pregnant women.

\section{Acknowledgements}

We thank Dr. Xue Jianjiang for her professional guide and support during the study. This study was sponsored by Chongqing Science-Health Union Medical Research Project (2018MSXM105).

\section{References}

[1] The death rate of Chinese pregnant women increased: or because that age-advanced women risked lives with second delivery

http://news.163.com/16/1006/00/C2LFF2DM00014JB5.html (accessed 13 November 2016).

[2] Kristoffersen AH., Petersen PH., RФraas T. and Sandberg S. (2017). Estimates of within-subject biological variation of protein $\mathrm{C}$, antithrombin, protein $\mathrm{S}$ free, protein $\mathrm{S}$ activity, and activated protein $\mathrm{C}$ resistance in pregnant women. Clinical Chemistry, 63 (4): 898-907.

[3] Parunov LA., Soshitova NP., Ovanesov MV., Panteleev MA. And Serebriyskiy II. (2015). Epidemiology of venous thromboembolism associated with pregnancy. Birth Defects Research part C Embryo Today, 105 (3): 167-184.

[4] Heavner MS., Mansouri R., Arndt M., Parkeret L. and Eyler RF. (2017). Thrombolysis for massive pulmonary embolism in pregnancy. Pharmacotherapy, 37 (11): 1449-1457.

[5] Othman M., McLintock C. and Kadir R.. (2016). Thrombosis and Hemostasis Related Issues in Women and Pregnancy. Seminars in Thrombosis and Hemostasis, 42 (7): 693-695.

[6] Olson JD. (2015). D-dimer: An Overview of Hemostasis and Fibrinolysis, Assays, and Clinical Applications. Advances in Clinical Chemistry, 69 (1): 1-6.

[7] Riva N., Vella K., Hickey K., Bertu L., Zammit D., and Spiteri S. (2018). Biomarkers for the diagnosis of venous thromboembolism: D-dimer, thrombin generation, procoagulant phospholipid and soluble P-selectin. Journal of Clinical Pathology, 71 (11): 1015-1022.

[8] Xie X., and Gou WL, Obstetrics and Gynaecology, 8th ed. People's Medical Publishing House: Beijing, 2014, pp. 221-2.

[9] Antony KM., Mansouri R., Arndt M., Hui SK., Jariwala P., and Mcmullen VM. (2015). Establishing thromboelastography with platelet-function analyzer reference ranges and other measures in healthy term pregnant women. American Journal of Perinatology, 32 (6): 545-554.

[10] Stanciakova L., Dobrotova M., Jedinakova Z., Duraj L., Skornova I. and Korinkova L. (2016). Monitoring of hemostasis and management of anticoagulant thromboprophylaxis in pregnant women with increased risk of fetal loss. Seminars in Thrombosis and Hemostasis, 42 (6): 612-621.

[11] Morikawa M., Yamada T., Akaishi R., Akaishi R., Koyama R. and Minakami H. (2011). Changes in D-dimer levels after cesarean section in women with singleton and twin pregnancies. Thrombosis Research, 128 (4): 33-38. 
[12] Sekiya A., Hayashi T., Kadohira Y., Shibayama M., Tsuda I., and Jin X. (2017). Thrombosis prediction based on reference ranges of coagulation-related markers in different stages of pregnancy. Clinical and Applied Thrombosis/ Hemostasis, 23 (7): 844-850.

[13] CLSI, Defining, establishing, and verifying of reference intervals in the clinical laboratory: Approved guideline, C28-A3E. 3rd ed. Wayne: Clinical and Laboratory Standards Institute, 2008, pp. 1-4.

[14] Gong JM., Shen Y. and He YX. (2016). Reference inervals of routine coagulation assays during the pregnancy and puerperium period. Journal of Clinical Laboratory Analysis, 30 (6): 912-917.

[15] Gutiérrez GI., Pérez CP., Martínez UJ., Izquierdo OG., Pérez MA., and Romualdo LG. (2018). D-dimer during pregnancy: establishing trimester-specific reference intervals. Scandinavian Journal of Clinical Laboratory Investigation, 78 (6): 439-442.

[16] Cui C., Yang S., Zhang J., WangG., Huang S. and Li A. (2017). Trimester-specific coagulation and anticoagulation reference intervals for healthy pregnancy. Thrombosis Research, 156 (1): 82-86. 